\title{
Excitation-Independent Emission of Carbon Quantum Dot Solids
}

\author{
Xuan-Dung Mai $\mathbb{D}^{1,2}$ Yen Thi Hai Phan, ${ }^{2,3}$ and Van-Quang Nguyen ${ }^{2}$ \\ ${ }^{1}$ Institute of Research and Development, Duy Tan University, Danang 550000, Vietnam \\ ${ }^{2}$ Department of Chemistry, Hanoi Pedagogical University 2, Xuan Hoa, Phuc Yen, Vinh Phuc, Vietnam \\ ${ }^{3}$ Archimedes Dong Anh Highschool, Dong Anh, Hanoi, Vietnam \\ Correspondence should be addressed to Xuan-Dung Mai; xdmai@hpu2.edu.vn
}

Received 10 August 2020; Revised 11 November 2020; Accepted 30 November 2020; Published 10 December 2020

Academic Editor: Dimitrios E. Manolakos

Copyright (C) 2020 Xuan-Dung Mai et al. This is an open access article distributed under the Creative Commons Attribution License, which permits unrestricted use, distribution, and reproduction in any medium, provided the original work is properly cited.

\begin{abstract}
Solid assemblies of carbon quantum dots (CQDs) are important for diverse applications including LEDs, solar cells, and photosensors; their optical and electrical properties have not been explored yet. Herein, we used amphiphilic CQDs synthesized from citric acid and thiourea by a solvothermal method to fabricate CQD solid films. Optical properties of CQDs studied by UVVis and photoluminescence spectroscopies indicate that CQDs possess three different emission centers at $425 \mathrm{~nm}, 525 \mathrm{~nm}$, and $625 \mathrm{~nm}$ originating from $\mathrm{C} \mathrm{sp}{ }^{2}$ states, N-states, and S-states, respectively. In a solid state, $\pi$ - $\pi$ stacking quenched the blue emission, while the red emission increased. Importantly, CQD films exhibited excitation independence, which is important to design solidstate lighting applications.
\end{abstract}

\section{Introduction}

Carbon quantum dots (CQDs) have been emerged as great photoluminescent nanomaterials for diverse applications including antibody labeling [1], heavy metal sensing [2], light-emitting diode (LED) lighting [3], and solar cells [4] owing to their low toxicity and large abundance. According to Yang's classification [5], CQDs include graphene QDs, which are similar to the mature graphene oxide, carbon polymer dots, and carbon nanodots. The latest type has been vastly reported and interchangeably called as CQDs owing to their simple synthesis and facile tunability in chemical composition and functionality [6, 7]. CQDs can be facilely synthesized by solvothermal methods using simple organic precursors such as citric acid, ethylenediamine, urea, thiourea, and phenylenediamine. In general, CQDs consist of polyaromatic hydrocarbons (PAHs) embedded in a carbogenic core and surface functional groups, which could include molecular-like fluorophores (MFs) and simple groups (SGs) such as $-\mathrm{NH}_{2},-\mathrm{COOH}$, $-\mathrm{OH}$, and $=\mathrm{CO}$. All components contribute to the optical properties of CQDs. Fu and coworkers proposed that PAHs of different sizes within the carbogenic core account for the excitation-dependent photoluminescence (PL) of CQDs [8]. The dependence of PL on the excitation wavelength is wildly observed in CQDs $[6,9,10]$, even on a single CQD [11], and it could be attributed to the existence of multiple emitters within CQDs $[12,13]$, especially to MFs [14-16]. On the contrary, Wang [17] and Yuan [18] groups reported that the emission spectra of CQDs, except for emission intensity, are independent on the excitation wavelength, probably due to simplicity in CQDs' structure [18].

In addition to studies on CQDs in solution or colloidal states, research studies on QD solids made of CQDs are strongly demanded because they are the state of application in real devices such as diode-type solar cells, organic lightemitting diodes (OLEDs), and photosensors. However, optical and electrical properties of CQD solids are not yet explored as compared with solids of other QDs such as $\mathrm{PbS}$ or CdTe QDs. There have been some reports demonstrating the effects of CQD aggregation on the absorption and emission of composites made of CQDs and polymers $[3,19] . \pi-\pi$ interaction in the solids enhances energy transfer among CQDs and is able to create new electronic states performing emission at long-wavelength regions 
[20-22]. Notably, in [21], the PL spectrum of colloidal CQDs exhibits strong excitation dependence in the UV to blue region, while that of solid CQDs shows fair excitation independence in the orange to red region. In a recent report, we demonstrated that both excitation and emission spectra of solid CQDs are red-shifted as compared with colloidal CQDs [22]. Herein, we prepared CQDs from a mixture of citric acid and thiourea by a solvothermal method. PL of colloidal CQDs exhibits solvent and excitation dependences in the blue to green region. Interestingly, the blue emission is dismissed in thin films of CQDs, which were prepared by a drop-casting method, while a new red emission appears at $630 \mathrm{~nm}$. Additionally, the emission of solid CQDs is excitation-independent. These findings are important to develop solid-state lightings.

\section{Experiment}

2.1. Materials. Chemicals including citric acid monohydrate (CA, 99.8\%, Aladdin Chemicals) and thiourea (TU, 99\%, Aladdin Chemicals) were purchased and used without any purification. A $50 \mathrm{ml}$ polypropylene (PPL) line in the autoclave was used to conduct solvothermal synthesis. A temperature-controlled electric oven was used to maintain the designed temperatures for the solvothermal syntheses.

2.2. Synthesis and Purification of CQDs. A mixture including $0.4156 \mathrm{~g}$ of CA and $0.76 \mathrm{~g}$ of TU dissolved in $35 \mathrm{ml}$ of acetone (HPLC grade, Aladdin Chemicals) was degassed by bubbling with nitrogen gas and transferred into an autoclave. The synthesis of CQDs was conducted at $180^{\circ} \mathrm{C}$ for 6 hours. After being cooled to room temperature, CQDs were precipitated by the addition of double distillated water and collected by centrifugation (at a speed of 8000 rounds per minute for 10 minutes at $5^{\circ} \mathrm{C}$ ). After three cycles of dispersion in acetone and precipitation with water, CQDs were finally dried under reduced pressure and stored at $5^{\circ} \mathrm{C}$ in the dark for further studies.

2.3. Preparation of CQD Solids. Quartz substrates $(2.5 \mathrm{~cm} \times 2.5 \mathrm{~cm})$ were sequentially washed with detergent solution, water, and ethanol. About $0.2 \mathrm{ml}$ of CQD solution $(20 \mathrm{mg} / \mathrm{ml}$ in acetone) was dropped onto a dried substrate at room temperature. Acetone solvent evaporated naturally leaving films of CQDs on the quartz substrate.

2.4. Characterizations. KBr pellet of CQDs was prepared for Fourier-transform infrared (FTIR) measurements, which were conducted on a Jasco FT/IR-6600 spectrometer. The absorption spectra of CQDs dissolved in various solvents were performed on a Shimadzu UV-2450 UV-visible spectrophotometer. Photoluminescence (PL) spectra of CQD solutions and films were carried out on a Nanolog spectrometer. Transmission electron microscopy (TEM) images of CQDs were obtained on a JEM-2100 microscope operating at $200 \mathrm{kV}$.

\section{Results and Discussion}

In TEM images of CQDs, as shown in Figure 1(a), CQDs could be resolved as dark and spherical dots with a diameter ranging from $2.5 \mathrm{~nm}$ to $8.5 \mathrm{~nm}$. The average diameter of CQDs was about $6 \mathrm{~nm}$, which is slightly bigger than the reported diameter of CQDs synthesized at $160^{\circ} \mathrm{C}$ [23]. It is because we synthesized CQDs at a higher solvothermal temperature, i.e., $180^{\circ} \mathrm{C}$. FTIR spectra of CQDs exhibited two broad absorption bands centered at $3358 \mathrm{~cm}^{-1}$ and $3213 \mathrm{~cm}^{-1}$, which could be assigned to stretching vibrations of $\mathrm{O}-\mathrm{H}$ and $\mathrm{N}-\mathrm{H}$ bonds. Multiple vibrational peaks at $3000-2850 \mathrm{~cm}^{-1}$ region could be attributed to $\mathrm{C}-\mathrm{H}$ stretching of aliphatic hydrocarbons or aldehydes. Two absorption bands peaking at $1800 \mathrm{~cm}^{-1}$ and $1700 \mathrm{~cm}^{-1}$ were assigned to the $\mathrm{C}=\mathrm{O}$ bond of anhydride structures, while three peaks at $1550 \mathrm{~cm}^{-1}, 1620 \mathrm{~cm}^{-1}$, and $1660 \mathrm{~cm}^{-1}$ were ascribed to amide (-CONH-) groups. Additionally, two peaks at $1450 \mathrm{~cm}^{-1}$ and $1350 \mathrm{~cm}^{-1}$ were attributed to stretching vibrations of $\mathrm{S}=\mathrm{O}$ and aromatic amine $\mathrm{C}-\mathrm{N}$ bonds $[6,20,23]$.

The absorption spectra of CQD solution in different solvents are shown in Figure 2(a). All the absorption spectra exhibit similar features with a shoulder at $350 \mathrm{~nm}$ and an absorption tail that extends to about $550 \mathrm{~nm}$. The shoulder could be assigned to $n-\pi^{*}$ electronic transitions of conjugated $\mathrm{C}=\mathrm{O}$ or $\mathrm{C}=\mathrm{N}$ bonds $[23,24]$. A weak absorption shoulder at about $450 \mathrm{~nm}$ could be seen, and it is reasonably attributed to the $n \longrightarrow \pi^{*}$ transition of the conjugated $\mathrm{C}=\mathrm{N}$ bonds [24]. Although the solvent causes negligible effects on the absorption properties of CQD solutions, Figure 2(a), the $\mathrm{PL}$ of CQDs changed significantly in the blue region (from $400 \mathrm{~nm}$ to $450 \mathrm{~nm}$ ) with the solvent as seen in Figure 2(b). At this moment, a detailed mechanism accounting for this blue quenching phenomenon is still ambiguous. Arshad and Sk studied the effects of the solvent on the emission of CQDs and pointed out that the aggregation of CQDs leads to enhancement in red emission and reduction in blue emission [20]. Probably, because CQDs absorb light significantly in the blue region (Figure 2(a)), CQD aggregation enhances energy transfer among CQDs so that emitted blue light was reabsorbed, while longer-wavelength light was less affected; this mechanism is known as Förster resonance energy transfer (FRET) [19, 22]. Alternatively, the blue emission originates mainly from $\mathrm{C} \mathrm{sp}{ }^{2}$ domains of CQDs, while the green emission (at c.a. $525 \mathrm{~nm}$ ) relates to surface $\mathrm{C}=\mathrm{N}$ states [24]. CQD aggregation caused by $\pi-\pi$ attraction among $C$ $\mathrm{sp}^{2}$ domains would quench the blue emission because excited carriers in $\mathrm{C} \mathrm{sp}^{2}$ are easily delocalized, while excited carries at surface states still stabilize via photorecombinations [21].

The effects of excitation on the emission of CQDs in the solution and solid state are summarized in Figure 3. Clearly, CQDs in the solution exhibited common excitation dependence, e.g., emission spectrum is red-shifted when the excitation wavelength increases $[6,9,11,25]$. On the contrary, PL spectra of CQD films exhibited similar features, with the exception of PL intensity, when varying the excitation wavelength. Compared to the PL spectrum of the 


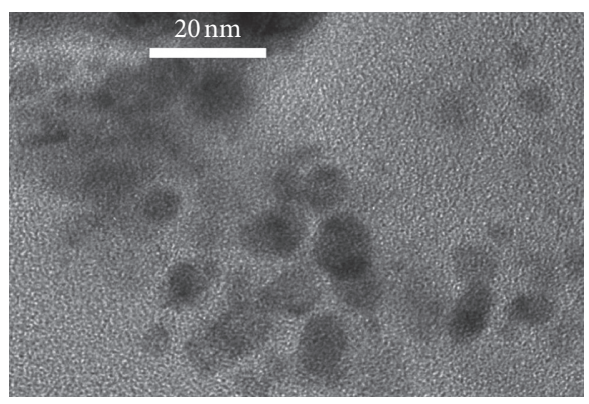

(a)

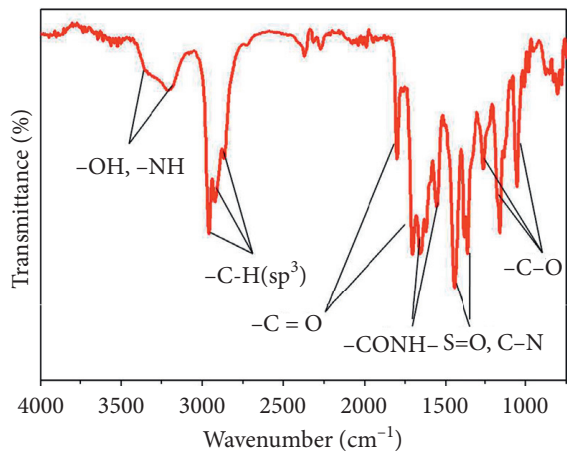

(b)

FIgURE 1: (a) TEM image and (b) FTIR spectrum of CQDs.

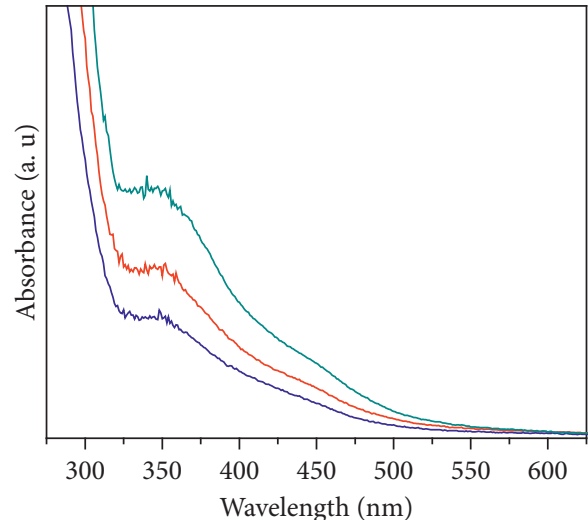

Solvents:

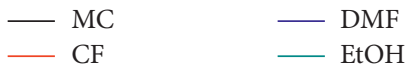

(a)

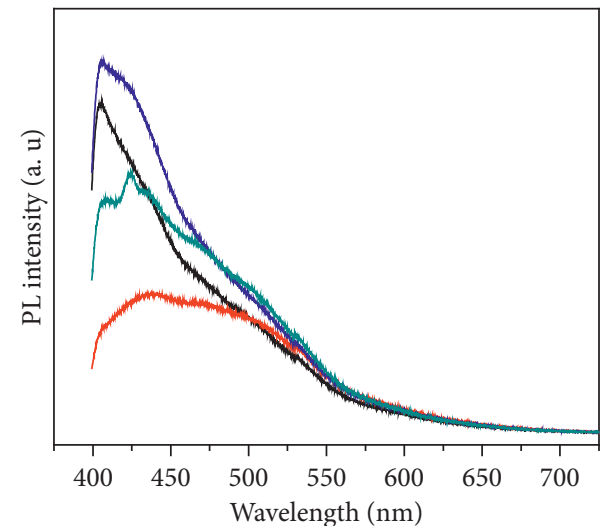

Solvents:

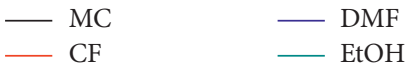

(b)

Figure 2: (a) Absorption and (b) PL (excited at $360 \mathrm{~nm}$ ) spectra of CQDs. MC: methylene chloride; CF: chloroform; DMF: dimethylformamide; EtOH: ethanol.

solution, a new emission center at $625 \mathrm{~nm}$ appeared, while blue emission at $425 \mathrm{~nm}$ disappeared. Apparently, under the UV lamp $(365 \mathrm{~nm})$, CQD solution exhibited blue-green color, while the CQD film showed yellow-red emission as seen in Figure 3. The PLE spectrum of CQDs in the solution monitored at $425 \mathrm{~nm}$ showed a broad excitation band around $355 \mathrm{~nm}$ (Figure 3). However, when excited near $355 \mathrm{~nm}$, CQD film did not emit at $425 \mathrm{~nm}$ but showed moderate-intensity emission at $525 \mathrm{~nm}$ and $625 \mathrm{~nm}$ as indicated by a low-intensity range in the PLE spectra of the CQD film in Figure 3. Additionally, the PLE spectra of the CQD film revealed a new excitation band centered at about $440 \mathrm{~nm}$.

To explain the emission of CQDs in a solid state, we adopted the model proposed by Qu et al. [24] and Yang et al. [21] as sketched in Figure 4. Blue emission $(425 \mathrm{~nm})$ originates mainly from $\mathrm{O}$-containing $\mathrm{C} \mathrm{sp}{ }^{2}$ domains, while green $(525 \mathrm{~nm})$ and red $(625 \mathrm{~nm})$ emissions are due to $\mathrm{N}$-state- and $\mathrm{S}$-state-assisted recombination. There is some degree of mixing between $\pi^{*}$ of $\mathrm{C} \mathrm{sp}{ }^{2}$ domains and O-states, between
$\mathrm{O}$-states and $\mathrm{N}$-states, and between $\mathrm{N}$-states and S-states. Therefore, excitation of CQDs in the solution at $340 \mathrm{~nm}$, which excites mainly $\mathrm{C} \mathrm{sp^{2 }}$ domains, results mainly in $\mathrm{O}$-state-related blue emission. When increasing the excitation wavelength, the possibility of the excited electron transferring from $\mathrm{O}$-states to $\mathrm{N}$-states increased, resulting in increasing emission intensity at $525 \mathrm{~nm}$ (Figure 3). Notably, red emission at $625 \mathrm{~nm}$ was still negligible in CQD solution when the excitation wavelength increased from $340 \mathrm{~nm}$ to $400 \mathrm{~nm}$. In a solid state, CQDs assembled via $\pi-\pi$ attraction, which enables electron transfer across CQDs (indicated by arrows in Figure 4(b)) and creates new electronic states of lower energy gap. The electron transferring among CQDs in the solid state accounts for negligible photoexcitation at wavelengths shorter than $325 \mathrm{~nm}$ where $\mathrm{C}$ sp $^{2}$ domains are excited (Figure 3) and mainly attributes to blue emission quenching. The existence of new electronic states induced by $\pi-\pi$ was deduced from the appearance of a new excitation band near $440 \mathrm{~nm}$ in PLE spectra of the CQD film (Figure 3). $\mathrm{N}$-states and S-states were reasonably unchanged by CQD 


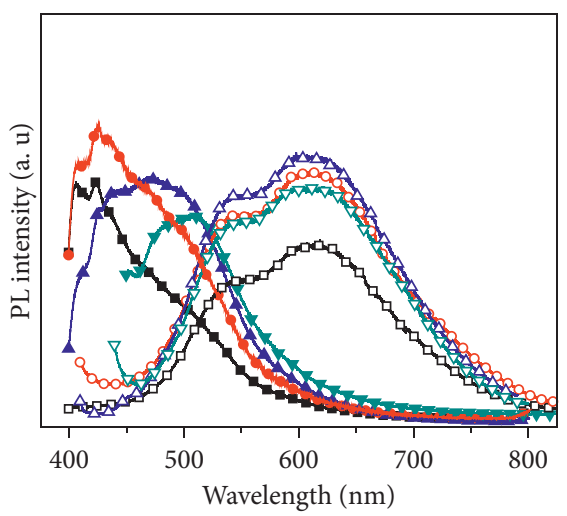

Excitation wavelength:

$\rightarrow 340 \mathrm{~nm} \longrightarrow 360 \mathrm{~nm}$

$\rightarrow 380 \mathrm{~nm} \quad \longrightarrow \quad 400 \mathrm{~nm}$

(a)

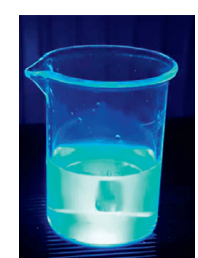

(b)

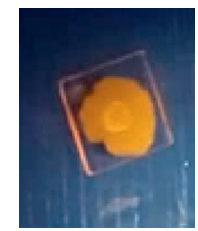

(c)

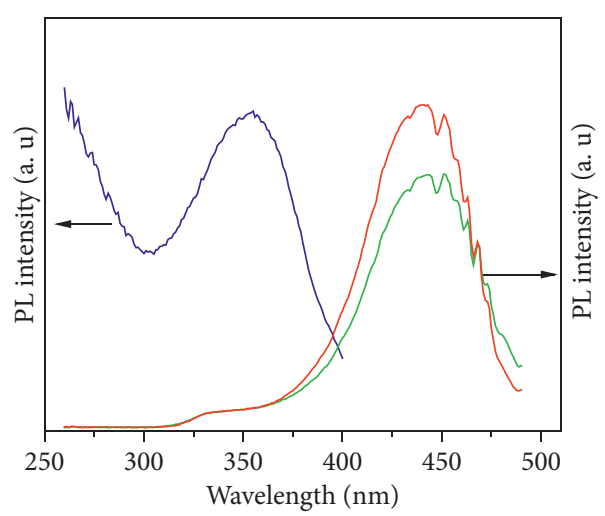

$425 \mathrm{~nm}(\mathrm{EtOH})$

$525 \mathrm{~nm}$ (film)

$625 \mathrm{~nm}$ (film)

FIgure 3: (a) PL spectra of CQD solution in EtOH (filled) and CQD film (open) excited at different excitation wavelengths. (b) and (c) Pictures of the CQD solution and film under the UV lamp $(365 \mathrm{~nm})$. (d) Normalized PLE spectra monitored at different wavelengths of CQDs dissolved in EtOH or in thin film.

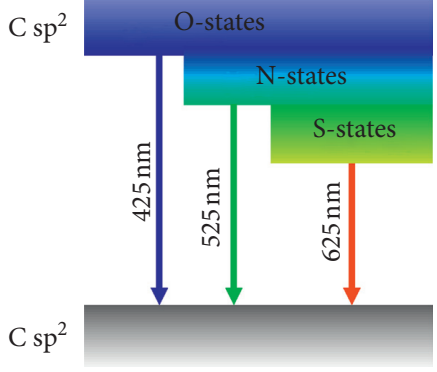

(a)

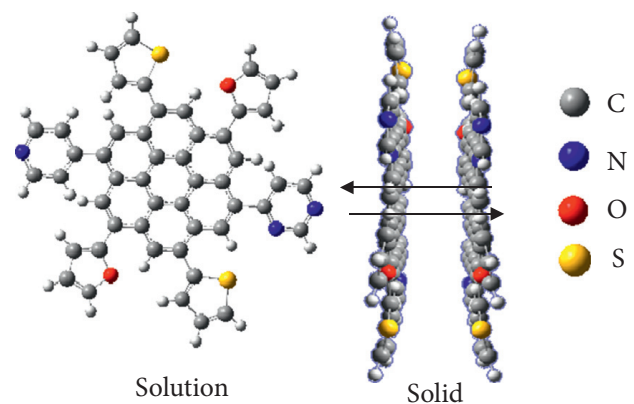

(b)

FIgure 4: (a) Plausible emission mechanism of the CQD solid. (b) Molecular representation of CQDs in the solution and solid state.

packing because $\mathrm{N}$ and $\mathrm{S}$ mainly involved in surface functional groups such as $-\mathrm{CONH},-\mathrm{NH}$, and $\mathrm{S}=\mathrm{O}$ (Figure $1(\mathrm{~b})$ ). The migration of electrons among CQDs in solids facilitated by $\pi-\pi$ packing would concentrate excited carriers to $\mathrm{N}$-states and S-states so that the green and red emissions were enhanced. Therefore, the green and red emissions were independent on excitation wavelength because $\mathrm{N}$ - and S-states were unaffected by $\pi-\pi$ stacking in the solid state, while it concentrated excited carriers to those states.

\section{Conclusion}

Spherical CQDs with a diameter ranging from 2.5 to $8.5 \mathrm{~nm}$ were successively prepared by solvothermal treatment of the mixture of citric acid and thiourea in acetone. While CQDs in the solution exhibited blue-green emission and excitationdependent photoluminescence, solid films of CQDs exhibited green-red emission, which were independent on excitation wavelength. Likely, green and red emission centers originated, respectively, from surface $\mathrm{N}$-states and
S-states, which were unaffected by $\pi-\pi$ stacking. The blue emission was quenched in a solid state because $\pi-\pi$ stacking induces electron transfer across CQDs. The independence of photoluminescence of CQD solids demonstrated herein makes designing excitation sources for solid-state lighting easier.

\section{Data Availability}

All the data used to support the findings of this study are available from the corresponding author upon request.

\section{Conflicts of Interest}

The authors declare that they have no conflicts of interest.

\section{Acknowledgments}

This research was funded by Hanoi Pedagogical University 2 via project no. C.2020-SP2-01. 


\section{References}

[1] M. X. Dung, N. T. Quynh, and T. Van Thao, "A Study on the use of Carbon Quantum dots on hCG immune analysis," TNU Journal of Science and Technology, vol. 225, pp. 58-64, 2020.

[2] M. X. D. Dang Thi Thu Huyen, N. Thi Quynh, L. Thi Hang, Le. Quang Trung, Do. Thi Thu Hoa, and P. Thi Hai Yen, "The synthesis of carbon polymer dot and its application in $\mathrm{Pb}$ (II) detection," TNU Journal of Science and Technology, vol. 189, pp. 45-51, 2018.

[3] Y. Zhang, P. Zhuo, H. Yin et al., "Solid-state fluorescent carbon dots with aggregation-induced yellow emission for white light-emitting diodes with high luminous efficiencies," ACS Applied Materials \& Interfaces, vol. 11, no. 27, pp. 24395-24403, 2019.

[4] X. Guo, H. Zhang, H. Sun, M. O. Tade, and S. Wang, "Green synthesis of carbon quantum dots for sensitized solar cells," ChemPhotoChem, vol. 1, no. 4, pp. 116-119, 2017.

[5] S. Zhu, Y. Song, X. Zhao, J. Shao, J. Zhang, and B. Yang, "The photoluminescence mechanism in carbon dots (graphene quantum dots, carbon nanodots, and polymer dots): current state and future perspective," Nano Research, vol. 8, no. 2, pp. 355-381, 2015.

[6] X.-D. Mai, T. Thi Kim Chi, T.-C. Nguyen, and V.-T. Ta, "Scalable synthesis of highly photoluminescence carbon quantum dots," Materials Letters, vol. 268, p. 127595, 2020.

[7] T. Q. Nguyen, T. T. Nguyen, T. M. Pham et al., "Universal method for preparation of metal-doped Carbon quantum dots," TNU Journal of Science and Technology, vol. 200, pp. 3-9, 2019.

[8] M. Fu, F. Ehrat, Y. Wang et al., "Carbon dots: a unique fluorescent cocktail of polycyclic aromatic hydrocarbons," Nano Letters, vol. 15, no. 9, pp. 6030-6035, 2015.

[9] Q.-B. Hoang, V.-T. Mai, D.-K. Nguyen, D. Q. Truong, and X.-D. Mai, "Crosslinking induced photoluminescence quenching in polyvinyl alcohol-carbon quantum dot composite," Materials Today Chemistry, vol. 12, pp. 166-172, 2019.

[10] T.-H. T. Dang, V.-T. Mai, Q.-T. Le, N.-H. Duong, and X.-D. Mai, "Post-decorated surface fluorophores enhance the photoluminescence of carbon quantum dots," Chemical Physics, vol. 527, p. 110503, 2019.

[11] B. van Dam, H. Nie, B. Ju et al., "Excitation-dependent photoluminescence from single-carbon dots," Small, vol. 13, no. 48, Article ID 1702098, 2017.

[12] S. Lai, Y. Jin, L. Shi, R. Zhou, Y. Zhou, and D. An, "Mechanisms behind excitation- and concentration-dependent multicolor photoluminescence in graphene quantum dots," Nanoscale, vol. 12, no. 2, pp. 591-601, 2020.

[13] H. A. Nguyen, I. Srivastava, D. Pan, and M. Gruebele, "Unraveling the fluorescence mechanism of carbon dots with sub-single-particle resolution," ACS Nano, vol. 14, no. 5, pp. 6127-6137, 2020.

[14] A. Sharma, T. Gadly, S. Neogy, S. K. Ghosh, and M. Kumbhakar, "Molecular origin and self-assembly of fluorescent carbon nanodots in polar solvents," The Journal of Physical Chemistry Letters, vol. 8, no. 5, pp. 1044-1052, 2017.

[15] J. Schneider, C. J. Reckmeier, Y. Xiong et al., "Molecular fluorescence in citric acid-based carbon dots," The Journal of Physical Chemistry C, vol. 121, no. 3, pp. 2014-2022, 2017.

[16] Y. Song, S. Zhu, S. Zhang et al., "Investigation from chemical structure to photoluminescent mechanism: a type of carbon dots from the pyrolysis of citric acid and an amine," Journal of Materials Chemistry C, vol. 3, no. 23, pp. 5976-5984, 2015.
[17] H. Wang, C. Sun, X. Chen et al., "Excitation wavelength independent visible color emission of carbon dots," Nanoscale, vol. 9, no. 5, pp. 1909-1915, 2017.

[18] F. Yuan, T. Yuan, L. Sui et al., "Engineering triangular carbon quantum dots with unprecedented narrow bandwidth emission for multicolored LEDs," Nature Communications, vol. 9, no. 1, p. 2249, 2018.

[19] D. Qu, D. Yang, Y. Sun, X. Wang, and Z. Sun, "White emissive carbon dots actuated by the H-/J-Aggregates and förster resonance energy transfer," The Journal of Physical Chemistry Letters, vol. 10, no. 14, pp. 3849-3857, 2019.

[20] F. Arshad and M. P. Sk, "Aggregation-induced red shift in N,S-doped chiral carbon dot emissions for moisture sensing," New Journal of Chemistry, vol. 43, no. 33, pp. 13240-13248, 2019.

[21] H. Yang, Y. Liu, Z. Guo et al., "Hydrophobic carbon dots with blue dispersed emission and red aggregation-induced emission," Nature Communications, vol. 10, no. 1, pp. 1-11, 2019.

[22] M. Van Tuan, L. T. Phuong, V. A. Duc, N. X. Bach, and M. X. Dung, "Enhanced energy transfer in carbon quantum dot solids," TNU Journal of Science and Technology, vol. 225, pp. 419-423, 2020.

[23] X. Miao, X. Yan, D. Qu, D. Li, F. F. Tao, and Z. Sun, "Red emissive sulfur, nitrogen codoped carbon dots and their application in ion detection and theraonostics," ACS Applied Materials \& Interfaces, vol. 9, no. 22, pp. 18549-18556, 2017.

[24] D. Qu, Z. Sun, M. Zheng et al., "Three colors emission from S,N Co-doped graphene quantum dots for visible light H2Production and bioimaging," Advanced Optical Materials, vol. 3, no. 3, pp. 360-367, 2015.

[25] M. X. Dung, M. V. Tuan, P. T. Long, and N. T. Mai, "Tuning the emission color of hydrothermally synthesized carbon quantum dots by precursor engineering," VNU Journal of Science: Natural Sciences and Technology, vol. 35, no. 1, 2019. 